\title{
Optical-fiber Electronic Speckle Pattem Interferometry for Quantitative Measurement of Defects on Aluminum Liners in Composite Pressure Vessels
}

\author{
Seong Jong Kim, Young June Kang*, and Nak-Jung Choi \\ Department of Mechanical Engineering, Chonbuk National University, Jeonju 664-14, Korea
}

(Received October 19, 2012 : revised December 27, 2012 : accepted December 28, 2012)

\begin{abstract}
Optical-fiber electronic speckle pattern interferometry (ESPI) is a non-contact, non-destructive examination technique with the advantages of rapid measurement, high accuracy, and full-field measurement. The optical-fiber ESPI system used in this study was compact and portable with the advantages of easy set-up and signal acquisition. By suitably configuring the optical-fiber ESPI system, producing an image signal in a charge-coupled device camera, and periodically modulating beam phases, we obtained phase information from the speckle pattern using a four-step phase shifting algorithm. Moreover, we compared the actual defect size with that of interference fringes which appeared on a screen after calculating the pixel value according to the distance between the object and the CCD camera. Conventional methods of measuring defects are time-consuming and resource-intensive because the estimated values are relative. However, our simple method could quantitatively estimate the defect length by carrying out numerical analysis for obtaining values on the $\mathrm{X}$-axis in a line profile. The results showed reliable values for average error rates and a decrease in the error rate with increasing defect length or pressure.
\end{abstract}

Keywords: Aluminum liner, Composite pressure vessel, Optic-fiber system, Defect length

OCIS codes : (060.0060) Fiber optics and optical communications; (060.2340) Fiber optics components; (120.0120) Instrumentation, measurement, and metrology; (120.2650) Fringe analysis

\section{INTRODUCTION}

A stable energy supply is indispensable for human life, given the fact that energy demand has been sharply increasing because of rapid industrial expansion. Extensive piping facilities are used in heat power plants and nuclear power plants, which supply most of the energy worldwide. Hence, there is a need to develop a system for avoiding breakage of pipes in piping facilities and to perform precise inspections of power facilities for defects to ensure a stable and continuous energy supply. When piping facilities involve extensive use of pipes and pressure vessels, defects from corrosion will be a common occurrence in such facilities. Such defects may often remain unnoticed until some destruction has been occurred [1].

The current techniques used for preventing such defects are ultra waves (microscopic defect detection and rapid detection is possible, but shape and internal structure of the product can affect the test), $\mathrm{x}$-rays (can be applied for all materials with different thickness defect detection but radiation safety management is challenging. More than 15 degrees rotated cracks cannot be detected and it is difficult to detect microcracks), and eddy currents (generally used for detecting defects in nonmagnetic materials and in the case of a magnetic object, identification of a defect is difficult because of residual magnetism). Although people use various ways to detect defects, there are different problems with the contact method, mainly lack of proper methodology and skill. Hence, to overcome these disadvantages, we have used the ESPI method in this work. The advantages of an ESPI system include rapid measurement, high accuracy, and non-destructive and non-contact inspection. Owing to such advantages, the ESPI method is assumed to stand above existing measurement methods across all industries. This optical method has been recently discussed regarding its use across many industries where they use non-destructive testing in their techniques [2].

An electronic speckle pattern interferometer can be classified, on the basis of the type of deformation that it can measure, into in-plane and out-of-plane. It can also be classified on the basis of the lengths of its components

\footnotetext{
*Corresponding author: yjkang@jbnu.ac.kr

Color versions of one or more of the figures in this paper are available online.
} 
and the shape of its optical components: a bulk-type and an optical-fiber type. Bulk-type ESPI of the optical configuration is complex and susceptible to outside influences (sensitive to light and vibration) [3]. Bulk-type ESPI is difficult to apply in the industrial field. In this study, we focused on the accurate measurement of defect length by using an optical-fiber-type ESPI. By preferring the optical-fiber-type ESPI over other ESPIs, we gain the following advantages; (1) It is cheaper than bulk-type ESPI (2) it is very simple to set up (3) there is no restriction on the type of material to be measured (4) complex components of existing measurement systems can be remodeled easily, and (5) it is less affected by disturbance and vibration than the bulk-type system $[3,4,5]$.

\section{THEORY}

We measured out-of-plane displacements by arbitrarily selecting two wavelengths that are superimposed on each other. Their condition, before and after the deformation of the specimen, is recorded through their four fringe images which are taken before and after their deformation by an external force $[6,8]$. The intensity of light, before the object is deformed by the force, is given as follows:

$$
I_{1}=I_{O}^{2}+I_{R}^{2}+I_{O} I_{R} \cos \left[\psi_{O}-\psi_{R}\right]
$$

In this equation, $I_{O}$ is the amplitude of the object beam and $I_{R}$ is the amplitude of one of the reference beams. $\Psi_{0}$, and $\Psi_{R}$ are the phases of the object and reference light beams, respectively $[7,8]$. When the object is deformed by the external force, the intensity of light is expressed as follows:

$$
\mathrm{I}_{2}=\mathrm{I}_{\mathrm{O}}^{2}+\mathrm{I}_{\mathrm{R}}^{2}+\mathrm{I}_{\mathrm{O}} \mathrm{I}_{\mathrm{R}} \cos \left[\psi_{\mathrm{O}}-\psi_{\mathrm{R}}+\Delta \psi\right]
$$

where, $\Delta \Psi$ denotes the phase variation in the object beam reflected from the position of deformation. For the relationship between $\mathrm{I}_{1}$ and $\mathrm{I}_{2}, 2 \pi$ and $(2 \mathrm{~N}+1) \pi$ are the maximum and minimum values of $\Delta \Psi$, respectively, and $\mathrm{N}$ is an integer [9].

The phase difference in the object beam before and after the deformation takes place is expressed as follows:

$$
\Delta \psi=2 \pi / \lambda\left\{\mathrm{d}_{1}(1+\cos \theta)+\mathrm{d}_{2} \sin \theta\right\}
$$

where $d_{1}$ represents the degree of deformation of the object in the direction of the wave front, $\mathrm{d}_{2}$ is the deformation in the $\mathrm{X}_{2}$ direction, and $\lambda$ is the wave length of the laser. Thus, the degree of deformation is expressed as follows:

$$
\Delta \mathrm{d}=\mathrm{n} \lambda /(1+\cos \theta)
$$

\section{EXPERIMENTAL SET-UP}

In this study, the method used for experimentation is the optical- fiber system which uses principles of out of the plane ESPI as shown in Fig. 2. The optical-fiber system is arranged on an optical table, which does not require protection against dust because it is not very sensitive to vibration and disturbance. A $532 \mathrm{~nm} \mathrm{CW}$ diode-pumped solid-state (DPSS) laser obtained from Cobolt Samba is used as a light source. The intensity of the laser was approximately $150 \mathrm{~mW}$. We used an optical-fiber system (Thorlabs Co.) having a dimension of $1 \mathrm{~m} \times 2 \mathrm{~m}$.

The permitted limit of the specific wavelength is between 532 and $640 \mathrm{~nm}$ and the laser beam emitted by the laser is divided into an object beam and a reference beam using a coupler with 50:50 magnifications. The object beam is emitted directly to a pressure vessel. The beam emitted to the pressure vessel enters a charge-coupled device (CCD) camera after reflecting from the surface of the pressure vessel. Then the reference beam is focused, and emitted into the CCD camera. The reference beam is controlled by a PZT cylinder, PT-130.14, obtained from Pi Co. An optical fiber is wound twenty times around the cylindrical

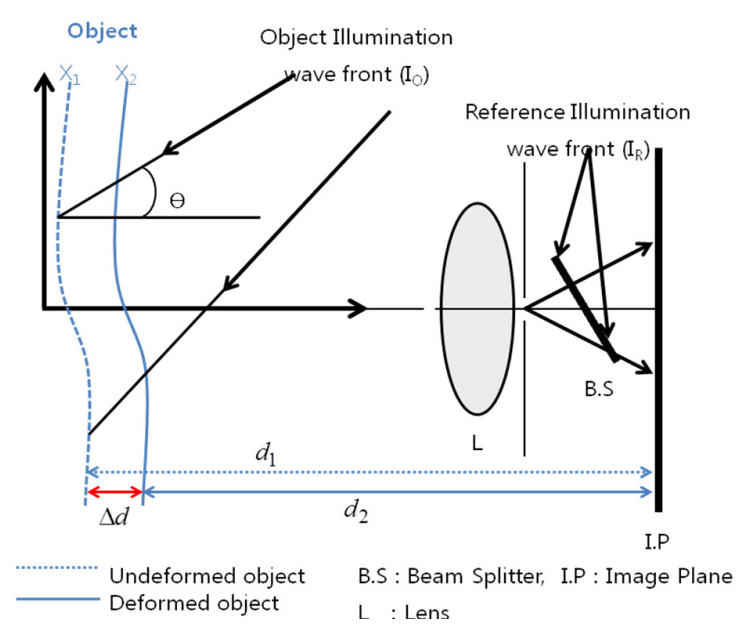

FIG. 1. Optical arrangement with fringes representing out-of-plane displacement.



FIG. 2. Configuration of optical-fiber ESPI. 
phase modulator, whose thickness can expand up to $\pm 16 \mu$ $\mathrm{m}$ when maximum voltage is applied. Thus the intensity distribution by interference is stored in a frame grabber through the CCD camera [7].

The frame grabber installed on a computer converts an analog signal to a digital one, and we used a frame grabber in our work because the data entered through the CCD camera was an analog signal. Interference fringes appear on the monitor after each of the eight images are extracted (four images, each captured both before and after deformation). They are saved on the image grabber. Eventually, the monitor displays a complete phase map according to the information of the displacement gradient using an equation that can estimate a value for the reference beam phase that is modulated four times using the PZT controller. Eventually, phase modulation to obtain a 4-bucket (4-step) algorithm uses PZT. Using the phase map obtained in this manner, we can understand the defect formation and estimate the displacement value $[9,10]$.

Out-of-plane deformation can be measured from the fringe pattern by phase. The size of the fringe pattern can be used as a measure of internal defect length. For quantitative measurement of the defect length, the size of the fringes that appears on the computer monitor should be known. Table 1 lists the lengths of fringes on the screen according to the distance between the specimen and the CCD camera. Calculations should be done in pixels in order to measure these lengths quantitatively. In the 15 -inch monitor used in this study, 640 pixels exist on a 12-inch width. Hence, on our monitor, 1 pixel was equivalent to $0.48 \mathrm{~mm}$. For example, Fig. 3 shows that when the distance between the specimen and the CCD camera is $80 \mathrm{~mm}$, an actual length of $30 \mathrm{~mm}$ appears to be $30 \mathrm{~mm}$ on the monitor.

However, we selected a distance of $400 \mathrm{~mm}$, which appeared as $60 \mathrm{~mm}$ on the monitor. In other words, the ratio of the actual distance to that appearing on the monitor is $1: 2$ (CCD $400 \mathrm{~mm}$ is $60 \mathrm{~mm}$ in monitor/ CCD $800 \mathrm{~mm}$ is 30 $\mathrm{mm}$ in monitor). To reduce error with decreasing distance, we took each measurement 2 times.

TABLE 1. Fringe lengths according to the distance at a length $30 \mathrm{~mm}$

\begin{tabular}{c|c|c}
\hline $\begin{array}{c}\text { Distance between } \\
\text { specimen and CCD } \\
\text { camera (mm) }\end{array}$ & $\begin{array}{c}\text { Value on monitor } \\
\text { (pixel) }\end{array}$ & $\begin{array}{c}\text { Value on monitor } \\
\text { (mm) }\end{array}$ \\
\hline 400 & 125.0 & 60.0 \\
\hline 500 & 105.0 & 50.4 \\
\hline 600 & 84.2 & 40.4 \\
\hline 700 & 73.0 & 35.0 \\
\hline 800 & 62.5 & 30.0 \\
\hline 900 & 56.3 & 27.0 \\
\hline 1000 & 52.1 & 25.0 \\
\hline 1100 & 45.8 & 22.0 \\
\hline 1200 & 41.7 & 20.0 \\
\hline
\end{tabular}

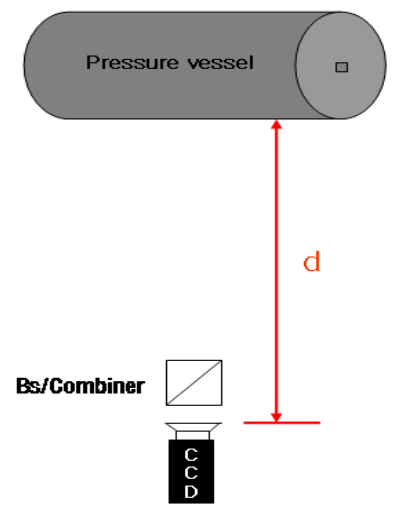

FIG. 3. Relationship between specimen and CCD camera.

\section{SPECIMEN AND TEST METHODS}

The specimen shown in Fig. 4 is made of aluminum 6061-T4 (Modulus of elasticity $=68.9 \mathrm{MPa}$, Poisson's ratio $=0.330$, Density $=2.70 \times 10^{-6} \mathrm{~kg} / \mathrm{mm}^{3}$ ). In our experiments, we used an aluminum liner which was a component of CNG fuel tanks used in buses as the specimen. The length, external diameter, and thickness of specimen were 1540 , 356 , and $5.5 \mathrm{~mm}$, respectively. Both ends of the specimen had screw threads which could be used to attach a cab that is used to apply the pressure to the specimen on the device. We can supply compressed gas to the specimen by creating a hole through the center of the cab. The surface of the specimen was painted white so that the aluminum liner could uniformly reflect an emitted laser beam. The internal defect in the specimen was circumferential having a depth of $3 \mathrm{~mm}$, and varying lengths of $10 \mathrm{~mm}, 20 \mathrm{~mm}$, and $30 \mathrm{~mm}$. To estimate the defect length, we used electric-spark machining to artificially create a defect inside the aluminum liner $[9,13]$.

Figure 5 shows the finite element analysis of the specimen. To ensure system stability during a pressure test, we used the simulation program ANSYS Workbench v.10. Mesh type was tetra mesh and element size: $10 \mathrm{~mm}$, the number of node: 90317 and element: 45947, material: aluminum alloy, analysis type: linear static analysis. To perform accurate measurements, the test pressure should not exceed the maximum allowable pressure. In our research, the displace-

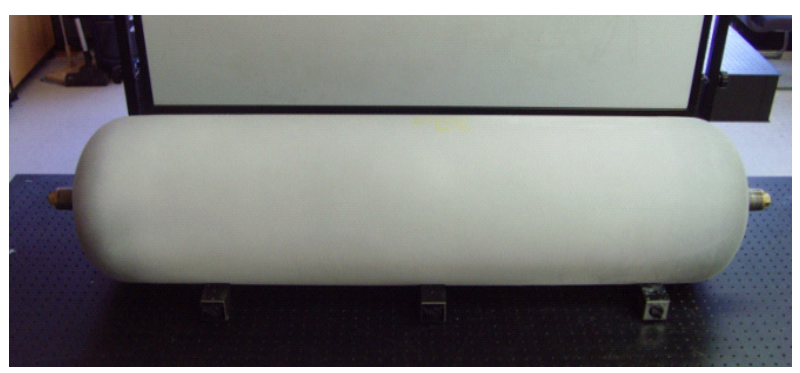

FIG. 4. Specimen image. 


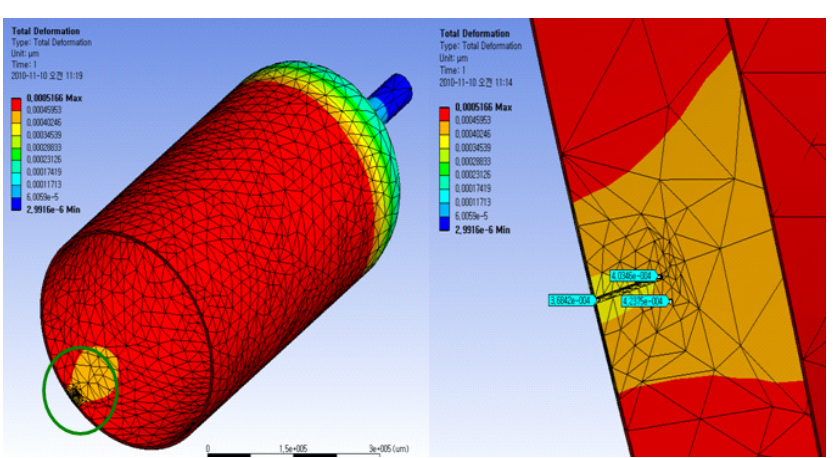

FIG. 5. Simulation image of displacement $0.070 \mathrm{Mpa}$.

ment of a defect in the pressure vessel was measured for a maximum pressure of $0.070 \mathrm{MPa}$ and minimum 0.014 $\mathrm{MPa}$. As shown in Fig. 5 the displacement of the defective part in the pressure vessel was about $0.0004 \mu \mathrm{m}$, and this value did not affect the estimated values.

\section{DATA EXTRACTION METHODS}

To analyze phase map data quantitatively, we used ESPI measurement equipment which is available commercially. It is called Q-100 Micro-star equipment, and it is used to measure small displacement. We used ISTRAMS 2.4.2 (4-bucket image processing analysis unit) to capture images of the defects to identify their shapes.

Through operation, we obtained a contour line with their standardized maximum and minimum. From the line profile data, we estimated the values of defect length using subtractions along the X-axis, as shown in Figs. 6 and 7.

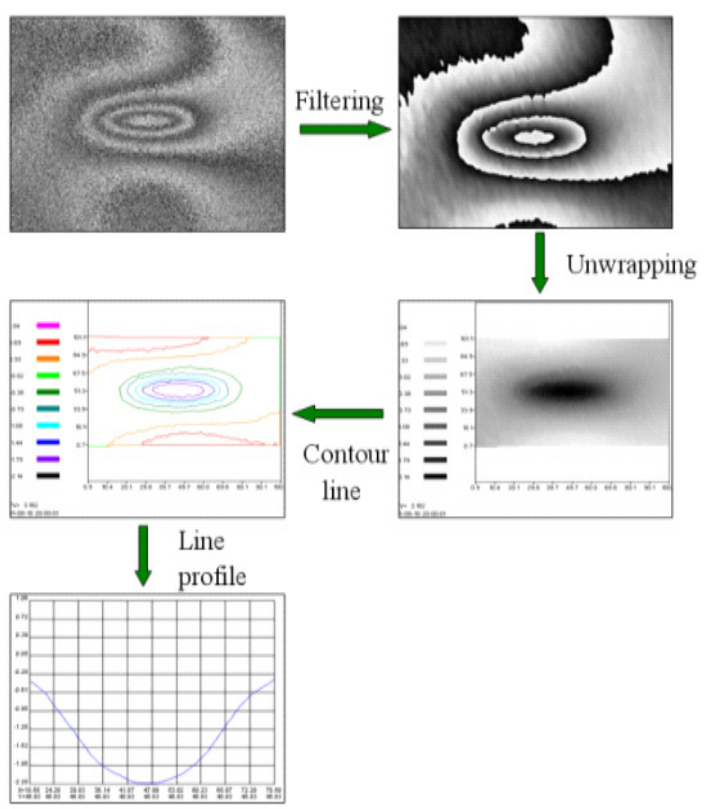

FIG. 6. Image processing.

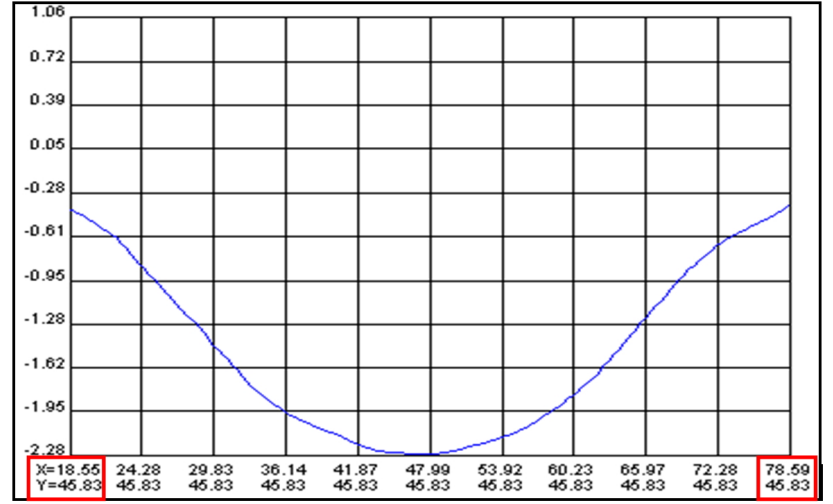

FIG. 7. Line profile at defect length of $30 \mathrm{~mm}(0.070 \mathrm{MPa})$.

\section{RESULTS}

Figure 8 shows the experimental results which indicate the phase map for the case of an axial flaw in the circumferential direction with a defect depth of $3 \mathrm{~mm}$ and three different defects lengths of 10,20 , and $30 \mathrm{~mm}$ using the optical-fiber ESPI system. The measurable laser-beam irradiation area is $144 \mathrm{~mm} \times 110 \mathrm{~mm}$ and the intensity of the laser used in these experiments was $150 \mathrm{~mW}$. We used a pressurizing method that input nitrogen gas into the pressure vessel to produce a deformation.

We applied a pressure of $0.014,0.028,0.042,0.056$, and $0.070 \mathrm{MPa}$ on specimens with three different defect lengths. To achieve accurate results, a pressure of 0.070 $\mathrm{MPa}$ was determined as the maximum pressure. If the pressure exceeded its maximum value, the interference fringe would have appeared overlapped with other fringes.

In this study, values of the defect length were estimated in the line profiling step in the four-step image processing

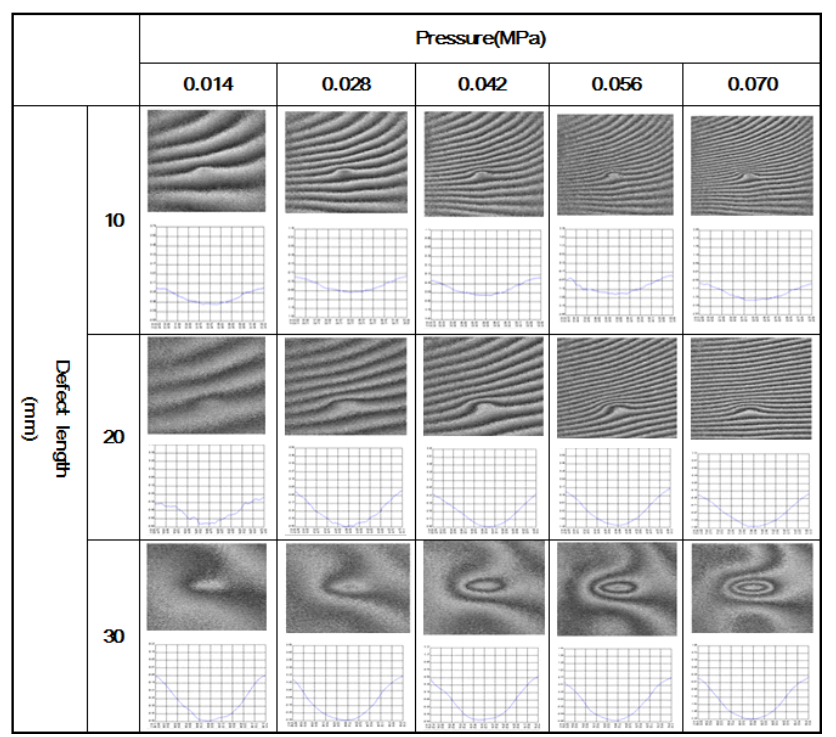

FIG. 8. Fringe pattern and Line profile obtained with each condition. 
TABLE 2. Estimated defect lengths for pressure of $0.070 \mathrm{MPa}$

\begin{tabular}{|c|c|c|c|c|}
\hline \multicolumn{5}{|c|}{ Pressure : $0.07 \mathrm{MPa}$} \\
\hline $\begin{array}{l}\text { Defect length } \\
(\mathrm{mm})\end{array}$ & $\begin{array}{c}\text { Estimated value } \\
\left(\mathrm{X}_{2}-\mathrm{X}_{1} / 2\right)\end{array}$ & $\begin{array}{l}\text { Error } \\
(\mathrm{mm})\end{array}$ & $\begin{array}{c}\text { Error rate } \\
(\%)\end{array}$ & $\begin{array}{c}\text { Average error } \\
(\mathrm{mm})\end{array}$ \\
\hline \multirow{10}{*}{10} & 10.105 & 0.105 & 1.05 & \multirow{10}{*}{0.058} \\
\hline & 10.055 & 0.055 & 0.55 & \\
\hline & 10.055 & 0.055 & 0.55 & \\
\hline & 10.040 & 0.040 & 0.40 & \\
\hline & 9.970 & -0.030 & 0.30 & \\
\hline & 9.925 & -0.075 & 0.75 & \\
\hline & 9.925 & -0.075 & 0.75 & \\
\hline & 10.055 & 0.055 & 0.55 & \\
\hline & 10.050 & 0.050 & 0.50 & \\
\hline & 10.040 & 0.040 & 0.40 & \\
\hline \multirow{10}{*}{20} & 20.075 & 0.075 & 0.38 & \multirow{10}{*}{0.046} \\
\hline & 20.055 & 0.055 & 0.28 & \\
\hline & 20.040 & 0.040 & 0.20 & \\
\hline & 20.030 & 0.030 & 0.15 & \\
\hline & 20.050 & 0.050 & 0.25 & \\
\hline & 20.075 & 0.075 & 0.38 & \\
\hline & 20.035 & 0.035 & 0.18 & \\
\hline & 20.035 & 0.035 & 0.18 & \\
\hline & 20.030 & 0.03 & 0.15 & \\
\hline & 20.035 & 0.035 & 0.18 & \\
\hline \multirow{10}{*}{30} & 30.020 & 0.020 & 0.07 & \multirow{10}{*}{0.031} \\
\hline & 30.035 & 0.035 & 0.12 & \\
\hline & 30.035 & 0.035 & 0.12 & \\
\hline & 30.000 & 0 & 0 & \\
\hline & 30.035 & 0.035 & 0.12 & \\
\hline & 29.945 & -0.055 & 0.18 & \\
\hline & 29.945 & -0.055 & 0.18 & \\
\hline & 30.035 & 0.035 & 0.12 & \\
\hline & 30.035 & 0.035 & 0.12 & \\
\hline & 30.000 & 0 & 0 & \\
\hline
\end{tabular}

equipment obtained from Microstar. This is a way to use maximum values and minimum values for the horizontal axis of the line profile. Through this study, our aim was to confirm the reliability of the proposed systems for accurately measuring defects inside the aluminum pressure vessels, as done in the case of the experimental specimen made of carbon steel.

To confirm the reliability of our proposed system, we compared and analyzed actual values with the estimated values. We performed experiments using the optical-fiber ESPI system over ten times. It should be noted that it is difficult to obtain a proper phase map that can provide the information about the defects inside a pressure vessel made of carbon steel under pressure conditions of less than

\section{$0.280 \mathrm{MPa}$.}

However, we obtained a good phase map for the defects on the aluminum liner at a substantially low pressure of 0.014MPa. A slightly larger error rate was estimated when the defect length and applied pressure were $10 \mathrm{~mm}$ and $0.014 \mathrm{MPa}$, respectively. However, it should be noted that as the defect length and applied pressure increased, the estimated values became remarkably consistent with the calculated one. Table 2 shows the values of defect lengths at a pressure of $0.070 \mathrm{MPa}$. The average errors for defect lengths 10, 20 , and $30 \mathrm{~mm}$ are $0.058,0.046$, and $0.031 \mathrm{~mm}$, respectively. This proves that the defect length increases with decreasing error rates. Fig. 9 shows a graph of the calculated values of defect lengths according to the pressure variation. 


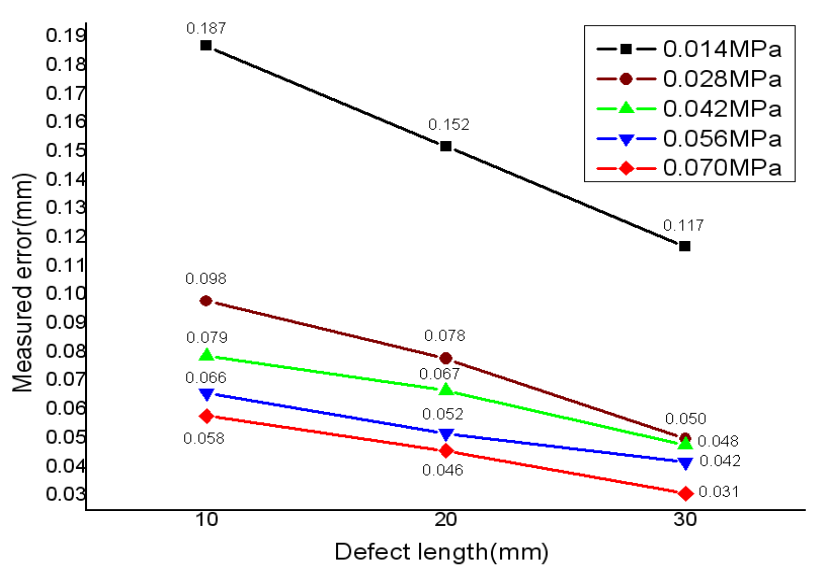

FIG. 9. Comparison of various estimated values for various values of pressure.

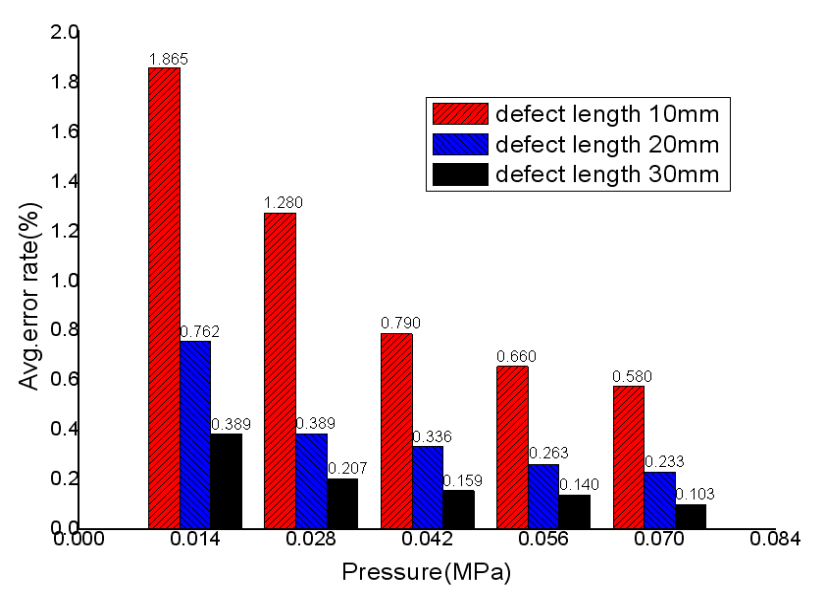

FIG. 10. Average error rate for each pressure.

In other words, the higher the pressure, the more the defect length error has been decreased. Fig. 9 is the average error rate; Fig. 10 showed the same pattern.

\section{CONCLUSION}

Our study proved that by using an optical-fiber ESPI system, it is possible to quantitatively measure the length of a defect on an aluminum liner used in composite pressure vessels. We verified the reliability of our proposed method after analyzing the experimental results.

We used a specimen made of aluminum in the experiments and designed the configuration and developed an optical measuring system for suitable use in the industry (seamless aluminum liner manufacturers). The new specimen is developed for making the composite pressure vessel that is installed on CNG buses. Currently, most buses in Korea use diesel fuel, but diesel is a major cause of air pollution. Hence, to ensure that these composite vessels are safe for real-time use, we determined the methods to quantitatively measure defects in composite pressure vessels that include aluminum liners.

In our experiments, we created defects on the specimen by electric- spark machining and measured these defects using our optical-fiber ESPI system. We estimated the defect lengths by indentifying them. We carried out a quantitative comparative analysis as shown in Table 2 . We found that the error rate decreased with increasing defect length or applied pressure.

We found that it is difficult to obtain a phase map that gives information about the defects inside the pressure vessel made of carbon steel under pressure conditions of less than $0.280 \mathrm{MPa}$. On the other hand, we obtained a good phase map for the pressure vessel consisting of an aluminum liner at a substantially smaller pressure of 0.014 MPa. However, when the pressure increased to more than $0.070 \mathrm{MPa}$, we did not obtain a suitable phase map because the fringe patterns overlapped each other in the process of filtering, which is one of the steps of four-step image processing. Hence, more deformations occurred in the aluminum liner than in the pressure vessel made of carbon steel under identical conditions, and thus, we determined $0.070 \mathrm{MPa}$ as the maximum test pressure. Furthermore, we calculated the increase in the defect length by using a finite element analysis program, ANSYS to estimate the corresponding increase in the error rate. The value of deformation was about $0.004 \mu \mathrm{m}$, and we confirmed that it did not affect the estimated values.

Through digital image processing, we estimated the defect per unit area by applying pressure on the aluminum liner. We were able to identify the position of the defect from the phase map and estimated the defect length by calculating the difference between the maximum and minimum values on the $\mathrm{X}$-axis in a line profile. In this research, we identified and measured only simple and artificial defects; however we intend to perform similar experiments on more complex defects. Through the Y-axis analysis of the line profile step, we will be able to estimate defect depth as well. Thus, aluminum liner is a difficult part of evaluation and certification standards. Future work can be applied to the evaluation certification through study data.

\section{ACKNOWLEDGMENT}

This research was supported by Basic Science Research Program through the National Research Foundation of Korea (NRF) funded by the Ministry of Education, Science and Technology (2010-0024952).

\section{REFERENCES}

1. K. S. Kim, W. S. Jang, and M. S. Hong, "Thermal strain analysis of composite materials by electronic speckle pattern interferometry," JMST 14, 477-482 (2000).

2. J. H. Yi, K. I. Lee, I. H. Park, and J. H. Kwon, "Laser 
beam shaping using hollow optical fiber and its application in laser induced thermal printing," J. Opt. Soc. Korea 13, 146-151 (2009).

3. D. Parisi, M. Facchini, and G. Beheim, "Automatic phase stepping in fiber-optic ESPI by closed-loop gain switching," IEEE T. Ins-Measur. 49, 823-828 (2000).

4. P. Thota, J. Leifer, S. W. Smith, and J. K. Lumpp, "Pattern evaluation for in-plane displacement measurement of thin films," Experimental Mechanics 45, 18-26 (2005).

5. X. Lï, J. H. Zhon, and D. D. Liu, "Application of color structured light pattern to measurement of large out-of-plane deformation," Acta Mechanica Sinica 27, 1098-1104 (2011).

6. H. M. Mourad and M. Y. A. Younan, "The effect of modeling parameters on the predicted limit loads for pipe bends subjected to out-of-plane moment loading and internal pressure," JPVT 122, 450-456 (2000).

7. R. Ritter, K. Galanulis, and D. Winter, "Notes on the application of electronic speckle pattern interferometry," Optics and Lasers in Engineering 26, 283-299 (1997).

8. K. Yohiaoui, D. G. Moreton, and D. N. Moffat, "Evaluation of limit load data for cracked pipe bends under opening bending and comparisons with existing solution," IJPVP 79, 27-36 (2002).
9. L. D. Melvin, B. A. Childers, and J. P. Fulton, "Quantitative analysis of a class of subsurface cracks using shearography and finite element modeling," Review of Progress in Quantitative Nondestructive Evaluation 12, 403-410 (1993).

10. W. Steinchen, L. X. Yang, M. Schuth, and G. Kupfer, "Electronic Shearography(ESPI) for direct measurement of strains," Proc. SPIE 2248, 210-221 (1994).

11. T. Suzuki, M. Matsuda, O. Sasaki, and T. Maruyama, "Laser-diode interferometer with a photothermal modulation," Appl. Opt. 38, 7069-7075 (1999).

12. P. Sandoz, T. Gharbi, and G. Tribillon, "Phase-shifting methods for interferometers using laser-diode frequency-modulation," Opt. Commun. 132, 227-231 (1996).

13. Prerana, R. K. Varshney, B. P. Pal, and B. Nagaraju, "High senstitve fiber optic temperature sensor based on a side-polished single-mode fiber coupled to a tapered multimode overlay waveguide," J. Opt. Soc. Korea 14, 337-341 (2010).

14. K. S. Kim, J. C. Jang, and J. H. Lee, "Effect of fiber dispersion and self-phase modulation in multi-channel subcarrier multiplexed optical signal transmission," J. Opt. Soc. Korea 14, 351-356 (2010).

15. J. Millerd, N. Brock, J. Hayes, M. Novak, and J. Wyant, "Pixelated phase-mask dynamic interferometer," Proc. SPIE 5531, 304-314 (2004). 